\title{
Identification of phenylpropanoid biosynthetic genes and phenylpropanoid accumulation by transcriptome analysis of Lycium chinense
}

Shicheng Zhao ${ }^{1}$, Pham Anh Tuan ${ }^{1}$, Xiaohua Li ${ }^{1}$, Yeon Bok Kim¹․ HyeRan Kim², Chun Geon Park ${ }^{3}$, Jingli Yang ${ }^{4}$, Cheng Hao Li ${ }^{4^{*}}$ and Sang Un Park ${ }^{1^{*}}$

\begin{abstract}
Background: Lycium chinense is well known in traditional Chinese herbal medicine for its medicinal value and composition, which have been widely studied for decades. However, further research on Lycium chinense is limited due to the lack of transcriptome and genomic information.

Results: The transcriptome of $L$. chinense was constructed by using an Illumina HiSeq 2000 sequencing platform. All 56,526 unigenes with an average length of 611 nt and an N50 equaling $848 \mathrm{nt}$ were generated from 58,192,350 total raw reads after filtering and assembly. Unigenes were assembled by BLAST similarity searches and annotated with Gene Ontology (GO) and Kyoto Encyclopedia of Genes and Genomes (KEGG) orthology identifiers. Using these transcriptome data, the majority of genes that are associated with phenylpropanoid biosynthesis in $L$. chinense were identified. In addition, phenylpropanoid biosynthesis-related gene expression and compound content in different organs were analyzed. We found that most phenylpropanoid genes were highly expressed in the red fruits, leaves, and flowers. An important phenylpropanoid, chlorogenic acid, was also found to be extremely abundant in leaves.
\end{abstract}

Conclusions: Using Illumina sequencing technology, we have identified the function of novel homologous genes that regulate metabolic pathways in Lycium chinense.

Keywords: Illumina sequencing, Lycium chinense, Phenylpropanoids, Chlorogenic acid

\section{Background}

Plant phenylpropanoids are a group of phenylalaninederived physiologically active secondary metabolites, such as lignins, flavonols, isoflavonoids, and anthocyanins [1]. They perform a vast array of important functions; for example, lignins reinforce specialized cell walls [2], flavonoids and isoflavonoids are involved in UV filtration [3,4] and symbiotic nitrogen fixation [5], and anthocyanins can protect plants against damaging photo-oxidative effects and UV irradiation [6]. Phenylpropanoids also have many beneficial functions for human health including anticancer [7] and anti-inflammatory properties [8].

\footnotetext{
*Correspondence: chli0@163.com; supark@cnu.ac.kr

${ }^{4}$ State Key Laboratory of Forest Genetics and Tree Breeding, Northeast Forestry University, 26 Hexing Road, Harbin 150040, China

'Department of Crop Science, Chungnam National University, 99 Daehak-ro, Yuseong-gu, Daejeon 305-764, Korea

Full list of author information is available at the end of the article
}

Phenylpropanoid biosynthesis starts with the formation of the aromatic amino acid phenylalanine. Phenylalanine ammonia lyase (PAL) catalyzes the phenylalanine into cinnamic acid. Cinnamate 4-hydroxylase (C4L) and 4-coumarate-CoA ligase (4CL) then catalyze the conversion of cinnamic acid to p-coumaroyl-CoA, which is the precursor for many phenylpropanoid products. Anthocyanins, flavonols and isoflaconoids are synthesized from p-coumaroyl-CoA through a complex phenylpropanoid pathway (Figure 1).

Lycium chinense, which is a member of the Solanaceae family, is a famous traditional Chinese herbal medicine that has a large variety of benefits, including anti-inflammatory effects [9], immunomodulating activity [10], anticancer properties [11], ability to reduce blood glucose and lipid levels [12], and anti-aging properties [13]. Researchers have isolated different secondary metabolites from L. chinense, such as alkaloids [14], carotenoids [15], 


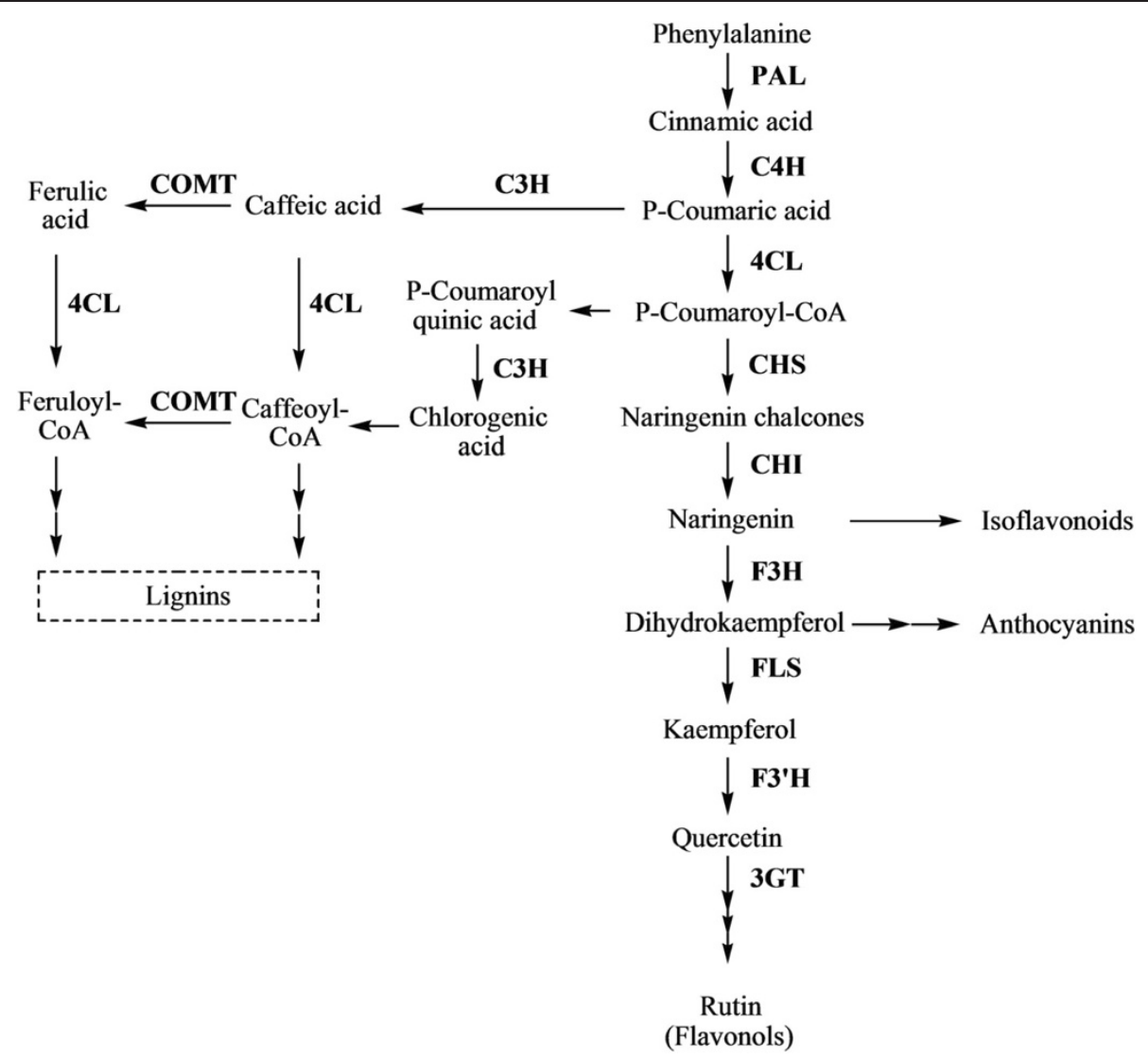

Figure 1 Schematic representation of phenylpropanoid biosynthesis in L. chinense. PAL, phenylalanine ammonium lyase; C4H, cinnamic acid 4-hydroxylase; 4CL, 4-coumarate-CoA ligase; CHS, chalcone synthase; CHI, chalcone isomerase; F3H, flavanone-3-hydroxylase; FLS, flavonol synthase; F3'H, flavonoid-3'-hydroxylase; 3GT, flavonoid 3-O-glucosyltransferase; C3H, p-coumarate-3- hydroxylase; and COMT, caffeate O-methyltransferase.

lignans [16], and betaine [17]. Partial sequences of specific genes have also been cloned in order to evaluate gene expression during the plant's development and to investigate the relationship of different genes in various plants $[18,19]$.

In recent years, next-generation sequencing (NGS) technologies such as 454 and Illumina platforms have been widely used in gene sequencing, most notably, in human genome sequencing [20,21]. Compared with traditional gene cloning, NGS technology has high efficiency (wholegenome sequencing), fast run times (ranging from hours to days) and high accuracy $[20,22]$. Among the different types of NGS technology, the Illumina Hiseq system has been widely used [23-25], owing to its high throughput, accuracy, and low costs.

The purpose of the current study was to investigate the phenylpropanoid biosynthetic pathway in $L$. chinense. Several full-length cDNAs encoding $P A L, C 4 H, 4 C L, C H S$, $\mathrm{C} 3 \mathrm{H}$, and $\mathrm{COMT}$, and partial-length cDNAs encoding CHI, F3H, FLS, F3' $H$, and $3 G T$ were identified and isolated. In addition, the relationship between the transcription levels of phenylpropanoid biosynthetic genes and phenylpropanoid accumulation were analyzed in different organs of $L$. chinense. To our knowledge, this study is the first to utilize transcriptome sequencing to investigate phenylpropanoid biosynthetic correlative gene expression in different organs of $L$. chinense.

\section{Results and discussion}

\section{Sequencing and sequence assembly}

Whole plantlet cDNA libraries were sequenced using the Illumina $\mathrm{HiSeq}^{\text {tm }} 2000$ system. After cleaning and quality checks, we obtained more than 54.1 million clean paired-end short reads of $90 \mathrm{nt}$ in length following sequencing (Table 1). The Q20 percentage (proportion of nucleotides with quality value larger than 20 in reads), $\mathrm{N}$ percentage, and GC percentage were $97.67 \%, 0.00 \%$ and $41.41 \%$, respectively. To facilitate sequence assembly, these reads were assembled using the Trinity program, resulting in 118,093 contigs with an average contig length of $312 \mathrm{nt}$ and an N50 of $511 \mathrm{nt}$, ranging from 200 nt to $>3,000$ nt (Table 1, Figure 2). Furthermore, Trinity 
Table 1 Summary of the transcriptome of $L$. chinense

\begin{tabular}{lllll}
\hline & Total number & $\begin{array}{l}\text { Total } \\
\text { nucleotides }(\mathbf{n t})\end{array}$ & $\begin{array}{l}\text { Mean } \\
\text { length (nt) }\end{array}$ & N50 \\
\hline Total raw reads & $58,192,350$ & - & - & - \\
Total clean reads & $54,138,216$ & $4,872,439,440$ & - & - \\
Total Contigs & 118,093 & - & 312 & 511 \\
Total unigenes & 56,526 & - & 611 & 848 \\
\hline
\end{tabular}

was used to assemble 56,526 unigenes with a mean size of $611 \mathrm{nt}$ and an N50 of $848 \mathrm{nt}$ (Table 1). The unigene size distribution showed the following: $14.25 \%(16,823)$ of the unigenes were between 500 and 1000 nt in length and $79.50 \%(93,885)$ were less than 500 nt long; $7.80 \%$ $(9,216)$ of contigs were between 1000 and $3000 \mathrm{nt}$, and $0.03 \%$ (32) were more than 3000 nt long (Figure 2).

\section{Unigene function annotation and pathways}

For annotation, the unigenes were further analyzed using BLASTX, on the National Center for Biotechnology Information (NCBI) website, against the non- redundant (nr) protein database with a cut-off E-value of $10^{-5}$; this resulted in the annotation of 34,684 unigenes $(61.36 \%$ of all 56,526 cleaned unigenes) (Table 2). The E-value distributions of the unigenes in the $\mathrm{Nr}$ database showed that $37.2 \%$ of the unigenes had strong similarity (smaller than $1 \mathrm{e}-60$ ), while the remaining $62.8 \%$ of the homologous sequences ranged from $1 \mathrm{e}-5$ to $1 \mathrm{e}-60$ (Figure $3 \mathrm{~A}$ ). The rates of the similarity distributions showed that $32.5 \%$ of the sequences had a similarity higher than $80 \%$, and $67.5 \%$ of the sequences had a similarity ranging from $19 \%$ to $80 \%$ (Figure 3B). The species distributions for the best match from each sequence are shown in Figure 3C. In detail, $34.07 \%$ of the unigenes had the highest homology to genes from Vitis vinifera, followed by Ricinus communis
Table 2 Summary of annotations of the $L$. chinense unigenes

\begin{tabular}{lll}
\hline & $\begin{array}{l}\text { Number of } \\
\text { blasted unigenes }\end{array}$ & Ratio \\
\hline All unigenes & 56,526 & - \\
Unigenes blasted against NR & 34,684 & $61.36 \%$ \\
Unigenes blasted against NT & 38,843 & $68.72 \%$ \\
Unigenes blasted against SWISS-PROT & 20,929 & $37.03 \%$ \\
Unigenes blasted against KEGG & 18,596 & $32.90 \%$ \\
Unigenes blasted against COG & 10,831 & $19.16 \%$ \\
Unigenes blasted against GO & 26,470 & $46.82 \%$ \\
All annotated unigenes & 42,022 & $74.34 \%$ \\
\hline
\end{tabular}

(12.47\%), Populus trichocarpa (11.35\%), Glycine max (7.21\%), Nicotiana tabacum (5.41\%), Solanum lycopersicum (5.38\%), Solanum tuberosum (3.77\%).

Other databases were also used to compare the unigenes, including 20,929 (37.03\% of all cleaned unigenes) sequences in SWISS-PROT, 18,596 (32.90\% of all cleaned unigenes) sequences in KEGG, 10,831 (19.16\% of all cleaned unigenes) sequences in Clusters of Orthologous Groups (COG), and 26,470 (46.83\% of all cleaned unigenes) sequences in Gene Ontology (GO) with the same identical cut-off E-value to supplement the annotations and functions. In total, 42,022 annotated transcripts were identified, representing approximately $74.34 \%$ of all cleaned unigenes (Table 2). Unigenes were compared with COGs in order to predict and classify their possible functions. The data comparison enabled the classification of 26 molecular families; the top category was "General function prediction only" (3,413 unigenes, 31.51\%) (Figure 4). For GO analysis, unigenes were divided into three major categories: biological processes, cellular components, and molecular function. Among the cluster of biological processes, cellular processes and metabolic processes were

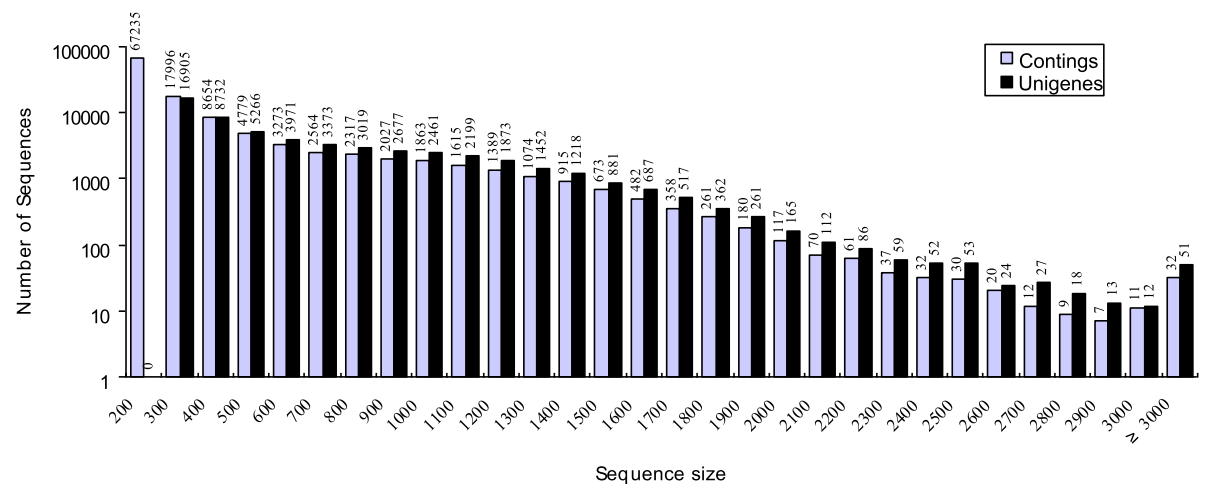

Figure 2 Length distribution of contigs and unigenes in L. chinense. 


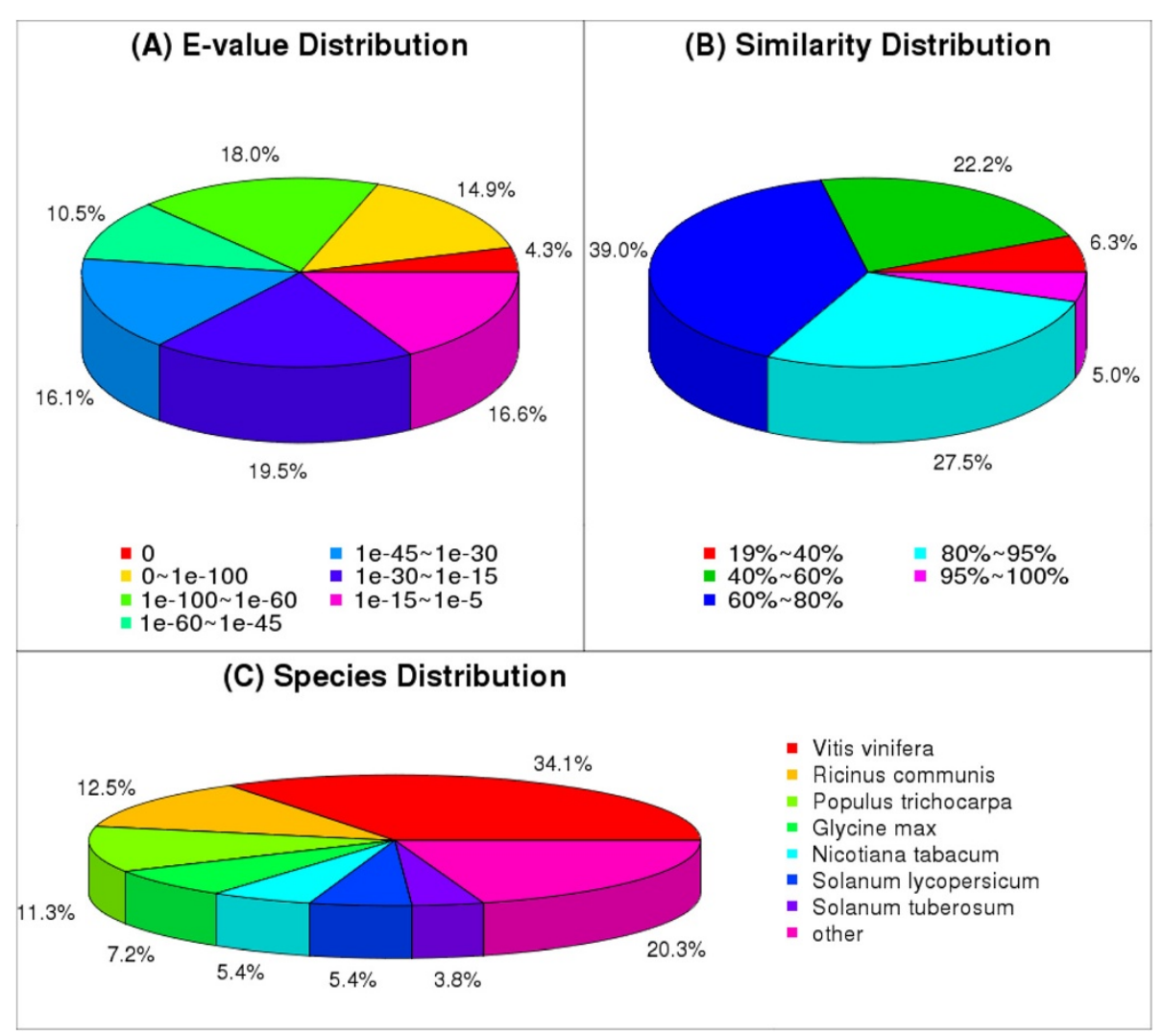

Figure 3 Figure of NR classification. (A) E-value distribution. (B) Similarity distribution. (C) Species distribution.

the two largest groups, containing 17,530 (66.23\%) and $17,089(64.56 \%)$ unigenes respectively. In the cellular component cluster, cells, cell parts, and organelles were dominant, containing 17,574 (66.39\%), 17,572 (66.38\%) and 13,141 (49.64\%) unigenes respectively. In the molecular function group, binding and catalytic activity were largest two sub-categories, containing 13,223 (49.95\%) and 13,422 (50.71) unigenes, respectively (Figure 5). All annotated unigenes were mapped to the KEGG database to define the cellular pathways containing these unigenes (Additional file 1). A total of 18,586 unigenes were assigned to 128 pathways. The most dominant clusters were metabolic pathways (4,489 unigenes, $24.14 \%)$, followed by biosynthesis of secondary metabolites $(2,459$ unigenes, $13.22 \%)$, plant hormone signal transduction $(1,088$ unigenes, 5.85\%), and plant-pathogen interaction (992 unigenes, 5.33\%).

Analysis of phenylpropanoid biosynthesis pathway genes from $L$. chinense unigenes

The sequences of phenylpropanoid biosynthesis pathway genes were identified in the NGS of the $L$. chinense database. They were confirmed for homology with the BLAST program and designed as LcPAL (719 amino acids), LcC4H (506 amino acids), Lc4CL (566 amino acids),
LcCHS (377 amino acids), LcCHI (184 amino acids), LcF3H (218 amino acids), LcFLS (246 amino acids), $L c F 3^{\prime} H$ (71 amino acids), Lc3GT (122 amino acids), $L c C 3 H$ (523 amino acids), and LcCOMT (378 amino acids). The data provided in Table 3 show that the phenylpropanoid biosynthetic genes from $L$. chinense exhibited high identity with other orthologous genes.

\section{Expression analysis of phenylpropanoid biosynthetic genes in different organs of $L$. chinense}

The expression of phenylpropanoid biosynthetic genes was analyzed in the roots, stems, leaves, flowers, green fruits, and red fruits of $L$. chinense by real-time PCR (Figure 6). LcPAL, the first enzyme in the phenylpropanoid biosynthetic pathway, was expressed at the highest levels in the flowers and green fruits, but was moderately expressed in the leaves, roots and red fruits, and only present at low levels in the stems. The expression patterns of $L c C 4 H, L c F 3^{\prime} H, L c 3 G T, L c C 3 H$, and $L c C O M T$ were similar, with observably higher expression in the red fruits than in the roots, stems, leaves, flowers, and green fruits. Among the phenylpropanoid biosynthetic genes of $L$. chinense, only $L c 4 C L$ was highly expressed in the roots, with similarly lower levels of expression in the other five organs. LcCHS exhibited high expression 

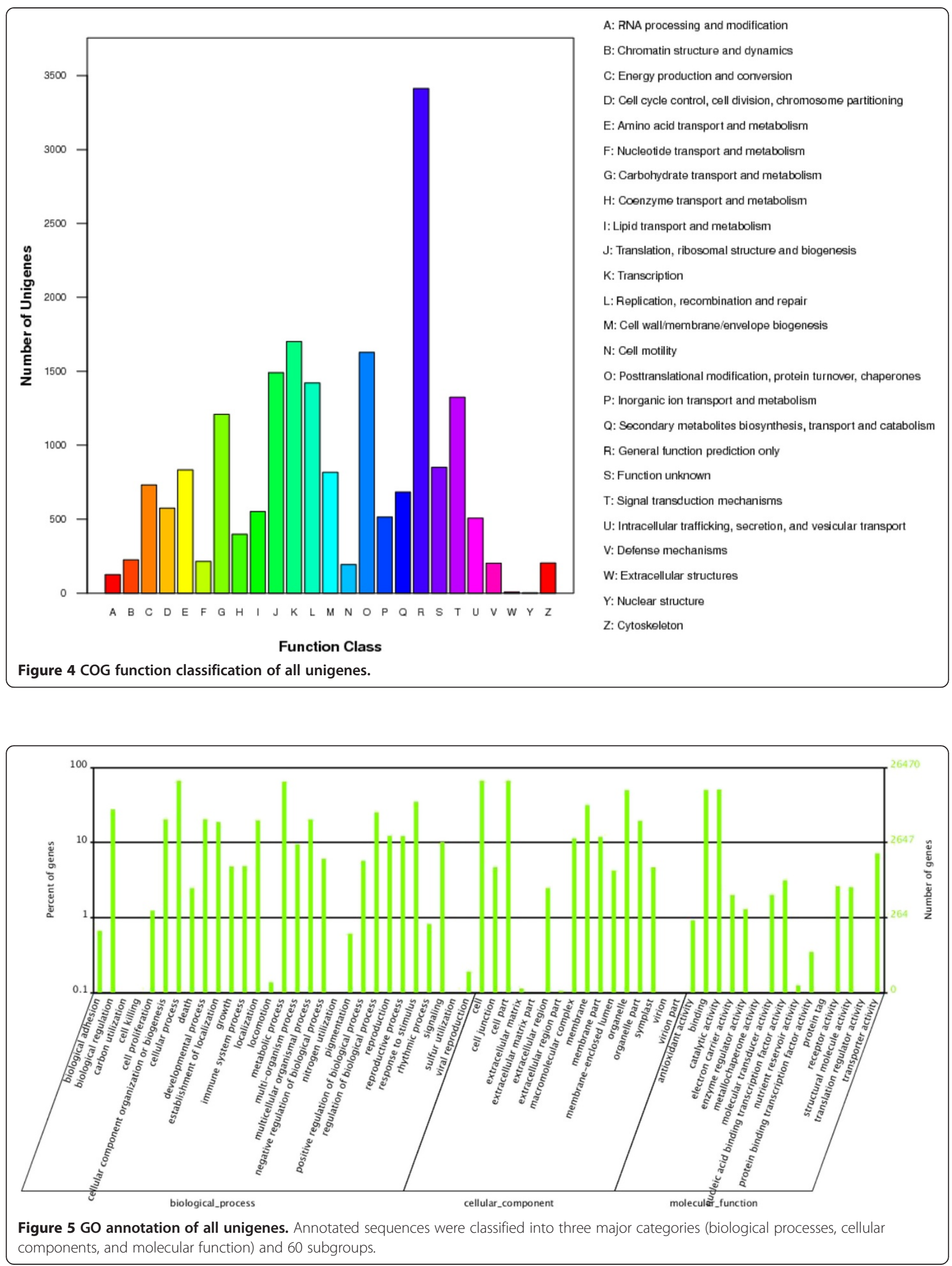
Table 3 Comparison of phenylpropanoid biosynthetic genes of $L$. chinense with the most orthologous genes

\begin{tabular}{|c|c|c|c|c|c|c|c|}
\hline \multirow{2}{*}{$\begin{array}{l}\text { Genes of } \\
\text { L. chinense }\end{array}$} & \multirow{2}{*}{$\begin{array}{c}\text { Length } \\
\text { (amino acid) }\end{array}$} & \multirow{2}{*}{$\begin{array}{l}\text { Orthologous genes } \\
\text { (Accession no.) }\end{array}$} & \multirow{2}{*}{$\begin{array}{l}\text { Identity } \\
(\%)\end{array}$} & \multicolumn{4}{|c|}{ (Continued) } \\
\hline & & & & LCCOMT & 378 & Capsicum annuum COMT & 84 \\
\hline \multirow[t]{3}{*}{ LCPAL } & 719 & Petunia axillaris PAL1 (JF793918) & 85 & & & (AF212316) & \\
\hline & & $\begin{array}{l}\text { Platycodon grandiflorus PAL2 } \\
\text { (JN392749) }\end{array}$ & 78 & & & $\begin{array}{l}\text { Capsicum chinense COMT } \\
\text { (AF081214) }\end{array}$ & 87 \\
\hline & & Coffea arabica PAL1 (JQ946534) & 76 & & & \multirow{2}{*}{$\begin{array}{l}\text { Catharanthus roseus COMT1 } \\
\text { (AY028439) }\end{array}$} & \multirow[t]{2}{*}{79} \\
\hline LCC4H & 506 & Capsicum annuum $\mathrm{C} 4 \mathrm{H}$ & 90 & & & & \\
\hline
\end{tabular}

Petunia hybrida $\mathrm{C} 4 \mathrm{H} 2$ (HM447145)

Lithospermum erythrorhizon $\mathrm{C} 4 \mathrm{H} 2$ (AB055508)

LC4CL $566 \quad$ Nicotiana tabacum 4CL1 (U50845) 89

Petunia hybrida 4CL (JN120849)

Solanum lycopersicum 4CL1 (XM_004235822)

LCCHS $\quad 377 \quad$ Solanum lycopersicum CHS
(XM_004239850)

Petunia hybrida CHS (X14592)

Senna alata CHS (AF358432)

LCCHI $\quad 184$

Nicotiana tabacum $\mathrm{CHI}$ (AB213651)

Capsicum annuum CHI (FJ705843)

Solanum lycopersicum $\mathrm{CHI}$ (XM_004238946)

$\begin{array}{lll}218 & \begin{array}{l}\text { Solanum tuberosum F3H } \\ \text { (AY102035) }\end{array}\end{array}$

Nicotiana tabacum F3H (AB289450)

Capsicum annuum $\mathrm{F} 3 \mathrm{H}$ (FJ705844)

$\begin{array}{lll}\text { LCFLS } & 246 \quad \begin{array}{l}\text { Solanum tuberosum FLS } \\ \text { (FJ770475) }\end{array}\end{array}$

Nicotiana tabacum FLS1 (DQ435530)

Solanum lycopersicum FLS (XM_004250281)

\begin{tabular}{|c|c|c|}
\hline \multirow[t]{3}{*}{ LCF3'H } & \multirow[t]{3}{*}{71} & $\begin{array}{l}\text { Solanum lycopersicum F3'H } \\
\text { (XM_004235959) }\end{array}$ \\
\hline & & Petunia hybrida F3'H (AF155332) \\
\hline & & $\begin{array}{l}\text { Antirrhinum majus F3'H } \\
\text { (DQ272592) }\end{array}$ \\
\hline \multirow[t]{3}{*}{ LC3GT } & \multirow[t]{3}{*}{122} & $\begin{array}{l}\text { Nicotiana tabacum 3GT } \\
\text { (AB723686) }\end{array}$ \\
\hline & & Petunia hybrida 3GT (AB027454) \\
\hline & & $\begin{array}{l}\text { Capsicum annuum 3GT } \\
\text { (JN808443) }\end{array}$ \\
\hline \multirow[t]{3}{*}{$\mathrm{LCC} 3 \mathrm{H}$} & \multirow[t]{3}{*}{523} & Coffea arabica C3H25 (JQ946542) \\
\hline & & $\begin{array}{l}\text { Populus trichocarpa C3H3 } \\
\text { (EU603301) }\end{array}$ \\
\hline & & $\begin{array}{l}\text { Trifolium pratense } \mathrm{C} 3 \mathrm{H} \\
\text { (GQ919201) }\end{array}$ \\
\hline
\end{tabular}

90

Table 3 Comparison of phenylpropanoid biosynthetic genes of $L$. chinense with the most orthologous genes (Continued)

levels in the red fruits and flowers, but lower levels in the other four organs. $\mathrm{LcCHI}, \mathrm{LcF} 3 \mathrm{H}$ and $\mathrm{LcFLS}$ were expressed at high levels in the leaves and flowers, at intermediate levels in the green and red fruits, and at very low levels in the roots and stems.

\section{Analysis of phenylpropanoid content in different organs of $L$. chinense}

The same plant materials used for quantitative real-time PCR were used for the HPLC analysis of phenylpropanoid accumulation. Trans-cinnamic acid, caffeic acid, ferulic acid, chlorogenic acid, kaempferol, and rutin were measured in the different organs of L. chinense (Figure 7). Small amounts of trans-cinnamic acid, caffeic acid, and ferulic acid were detected in the roots, stems, green fruits, and red fruits. In flowers, all of the identified compounds were very found to be present at high levels. The leaves contained abundant amounts of chlorogenic acid $(12,181.98 \mu \mathrm{g} / \mathrm{g}$ dry weight), caffeic acid $(334.85 \mu \mathrm{g} / \mathrm{g})$, and rutin $(56.14 \mu \mathrm{g} / \mathrm{g})$. High amounts of rutin were also found in green fruits $(43.53 \mu \mathrm{g} / \mathrm{g})$, flowers $(35.99 \mu \mathrm{g} / \mathrm{g})$, and stems $(31.54 \mu \mathrm{g} / \mathrm{g})$.

LCPAL, which carries out the first catalysis step in the phenylpropanoid biosynthesis pathway, was highly expressed in flowers in L. chinense, and the metabolite of this gene, cinnamic acid, was correspondingly the highest in flowers. The gene expression analysis showed that the expression level of $L c P A L$ in green fruits was also high; however, the content of its metabolites in green fruits was very low. This is probably owing to the fact that PALs are codified by a multigene family in plants [26,27], suggesting the existence of other $P A L$ isoforms in green fruits. In previous study of our laboratory, the expression of $\mathrm{C} 4 \mathrm{H}$ in different organs was diverse between different species. In plant Allium. sativum, the highest expression level occurred in the root, but in plant Agastache. rugosa, ArC4H was expressed highest in flowers [28,29]. In L. chinense, we found that the expression levels of $\mathrm{LcC} 4 \mathrm{H}$ in red fruits was significantly higher than that that in other organs. $L c 4 C L$ was highly expressed in root, this result is consistent with our laboratory's previous study in tartary buckwheat Hokkai T10 [30]. But in L. chinense's roots, the expression of $L c 4 C L$ was significantly higher than other 


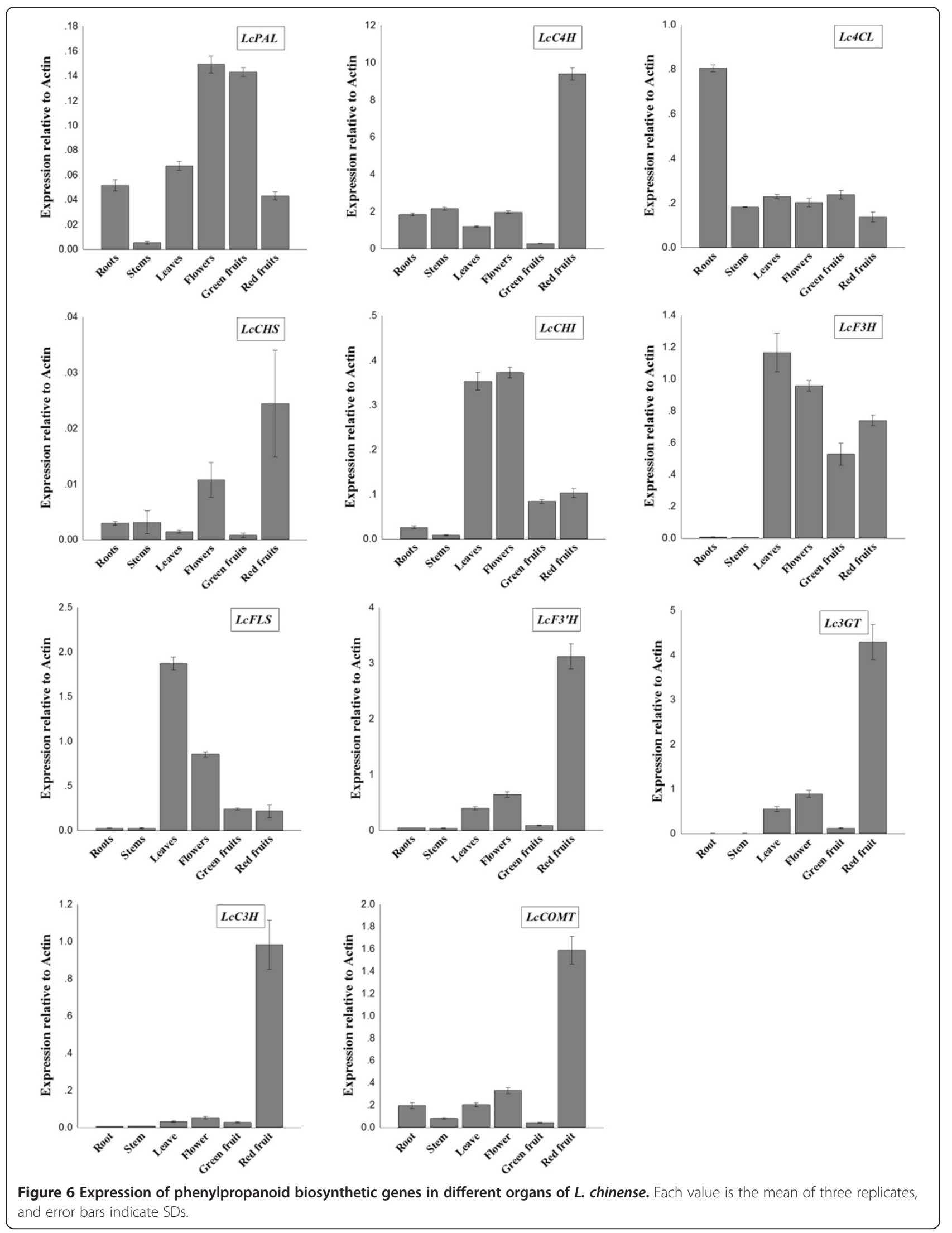




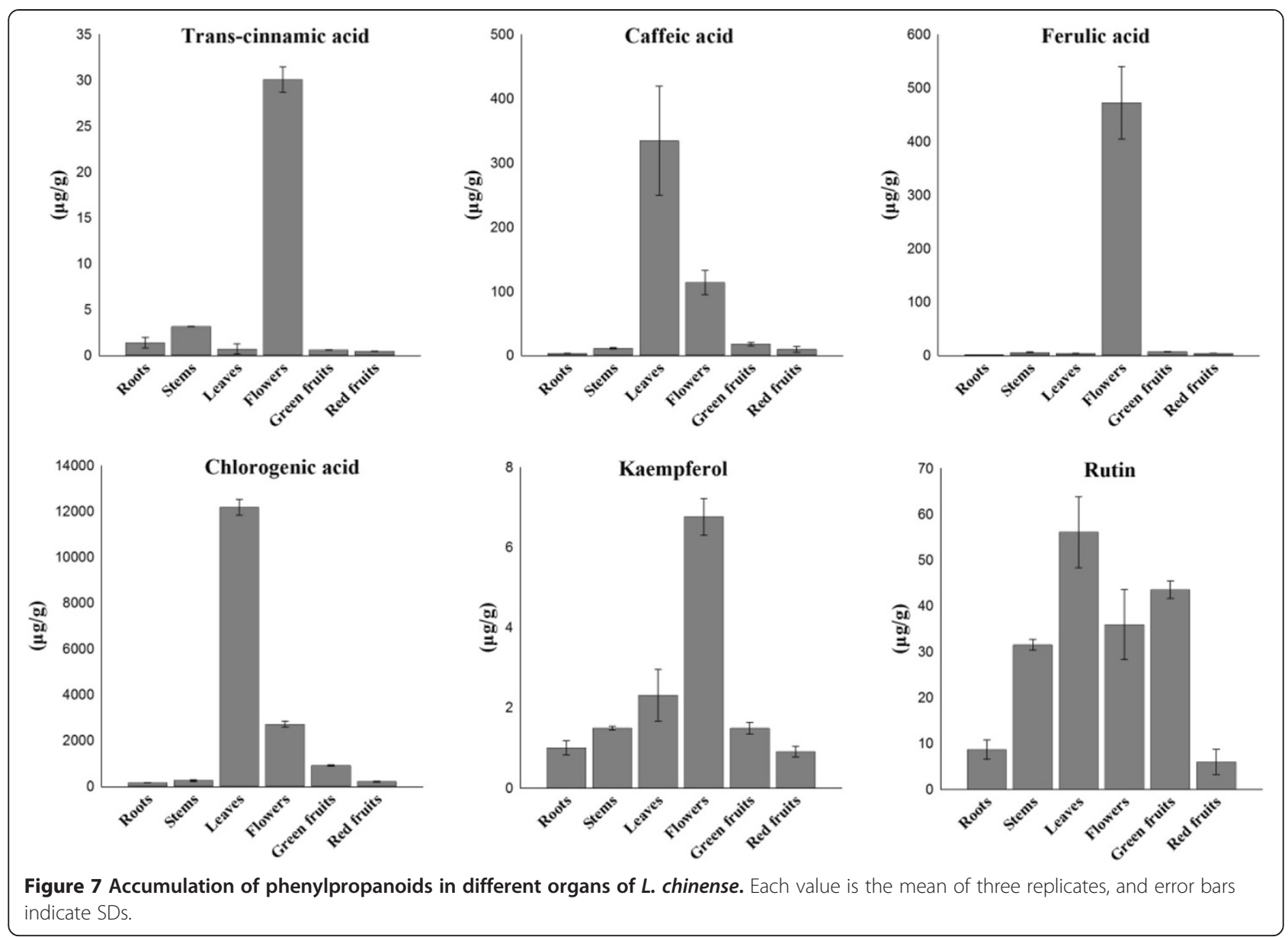

organs. In our laboratory's previous study, they also found two isoform FLS genes in tartary buckwheat, and that two FLS gene expressions were different in different organs [31]. This explains why the expression of the LcFLS was inconsistent with that of phenylpropanoid product, kaempferol. To content of kaempferol in L. chinense, it's much similar with that in buckwheat, highly accumulate in flowers than other organs. We found that the expression levels of $L c C 4 H, L c C H S, L c F 3^{\prime} H, L c 3 G T, L c C 3 H$, and $L c C O M T$ in red fruits was significantly higher than that in other plant organs but that the levels of the corresponding phenylpropanoids in red fruits was lower. It is well known that phenylpropanoid biosynthesis is a very complex process and that multiple enzyme metabolites are produced. Moreover, some enzymes exist in many isoforms. Therefore, we felt it was not appropriate to relate gene expression and metabolite production absolutely. As shown in Figure 7, the content of caffeic acid and chlorogenic acid in different organs has an essentially similar trend, where it is highest in leaves, followed by flowers; lower levels are present in other organs. In the phenylpropanoid biosynthesis pathway (Figure 1), caffeic acid and chlorogenic acid are regulated enzymes encoded by the same gene, $\mathrm{C} 3 \mathrm{H}$.

In the current study, the content of chlorogenic acid was found to be very high in leaves $(12,181.98 \mu \mathrm{g} / \mathrm{g})$. Chlorogenic acid is a polyphenol compound found in plants and animals and has many biological functions, including antioxidant [32], anti-stress [33], and antiviral [34] properties. Dong et al. [35] investigated the impact of selenium on the content of chlorogenic acid in leaves of L. chinense; the study found that the chlorogenic acid content $(10,846 \mu \mathrm{g} / \mathrm{g})$ in the presence of $0.01 \mu \mathrm{g} / \mathrm{g}$ sodium selenite was much higher than that in the control plants $(78.36 \mu \mathrm{g} / \mathrm{g})$. Therefore, we speculate that there were trace amounts of selenium at the experimental farm from which the $L$. chinense plants used in this study were derived. The presence of environmental elements, such as selenium, may produce certain effects on other phenylpropanoids [36]. In this stdy, we found most phenylpropanoids were accumulate in flowers and leaves, some phenylpropanoids such as kaempferol and rutin also have high accumulate in stems and green fruits,but the content of phenylpropanoids were lowest in roots and red fruits. Nowadays, most 
consumer purchase fresh or dry red fruits as part of diet. As a reault, we suggest consumer to use other parts of $L$. chinense to maximize the health benefits of phenylpropanoids.

\section{Conclusions}

In this study, we developed a rapid and cost-effective method for transcriptome analysis of Lycium chinense using Illumina sequencing technology. Using the annotation, we found many transcripts that encode for putative genes that are involved in phenylpropanoid biosynthesis. But not all genes were found, that mostly because the sample which used for RNA sequencing was not the stage that all genes were expressed. To extensively study the genes related the biosynthesis of secondary metabolites, we have plan to use other tissues of L.chinense for RNA sequencing in future. We also compared gene expression in different organs. We noticed that most phenylproanoid genes were highly expressed in the leaves, flowers, and red fruits. Our compound analysis indicated that a number of phenylpropanoids were present in the leaves and flowers of L. chinense, such as chlorogenic acid, caffeic acid, and rutin.

\section{Methods}

\section{Plant material and RNA extraction}

Plant materials were collected from Lycium chinense cutting seedling grown outdoor at an experimental farm at Chungnam National University for 1 year (Daejeon, Korea). Whole plantlets induced from the twig segment of L. chinense were used for transcriptome analysis [37]. Different organs from the mature plant, including the roots, stems, leaves, flowers, and fruits from two different stages of maturation (green fruits and red fruits), were excised. All samples were immediately frozen in liquid nitrogen and then stored at $-80^{\circ} \mathrm{C}$ and/or freezedried for RNA isolation and/or high-performance liquid chromatography (HPLC) analysis. The $L$. chinense samples were ground into powder in a mortar with liquid nitrogen, and total RNA was isolated separately using the RNeasy Plant Mini kit (Qiagen, Valencia, CA).

\section{Illumina sequencing}

Beads with Oligo (dT) were used to isolate poly (A) mRNA after total RNA was extracted. Fragmentation buffer was then added to digest the mRNA into short fragments. Using these short fragments as templates, random hexamer primers was used to synthesize the first-strand cDNA. The second-strand cDNA was then synthesized using buffer, dNTPs, RNase $\mathrm{H}$, and DNA polymerase I. Short fragments were purified with a QiaQuick PCR extraction kit and resolved with EB buffer for end reparation and addition of poly (A). Following this, the short fragments were connected with sequencing adapters and analyzed by agarose gel electrophoresis in order to select suitable fragments for amplification by PCR. The resulting cDNA library was then sequenced using an Illumina HiSeq $^{\text {ma }} 2000$ system. Illumina Sequencing was performed at the Beijing Genomics Institute (BGI) genomic Center in Shenzhen, China (http://www.genomics.cn).

\section{Reads filtration and assembly}

Image data output from the sequencer was transformed by base calling into sequence data, also called raw data or raw reads. Before the assembly, the raw reads contain filtered reads and have adapters, a proportion of unknown nucleotides larger than $5 \%(\mathrm{~N} \geq 5 \%)$, duplication sequences, and low quality bases (more than $20 \%$ nucleotides with quality value $\leq 10$ ), which negatively affect the subsequent bioinformatics analysis. Therefore, dirty raw reads were removed. The clean reads were then assembled using Trinity software (release-20120608) [38]. Trinity first combines reads with a certain length of overlap to form longer fragments, which are called contigs. The reads are then mapped back to the contigs. Finally, Trinity connects the contigs and gets sequences that cannot be extended on either end. Such sequences are defined as unigenes. Unigenes from each sample's assembly can be used in further processes of sequence splicing and redundancy removal with sequence clustering software in order to acquire non-redundant unigenes that are as long as possible. The sequencing data from this study were deposited in the NCBI Sequence Read Archive (SRA, http://www.ncbi.nlm.nih.gov/Traces/sra/) under accession number SRR886280.

\section{Unigene function annotation}

Information was obtained regarding functional annotation, including protein functional annotation, COG functional annotation, and GO functional annotation of unigenes. Unigene sequences were first submitted to protein databases for alignment and comparison by BLASTX algorithms with a significant threshold of E-value $\leq 10^{-5}$, like nr, SWISS-PROT, KEGG, and COG. In addition, unigenes were aligned by Blastn to nucleotide databases nt (E-value $\left.\leq 10^{-5}\right)$, retrieving proteins with the highest sequence similarity with the given unigenes along with their protein functional annotations. In addition, the orientation of Illumina sequences that could not be obtained directly from sequencing were derived from BLAST annotations (ftp://ftp.ncbi.nih.gov/blast/executables/blast+/LATEST/). For other sequences not involved in the BLAST search, we used the ESTScan program (version 3.0.2, http://www. ch.embnet.org/software/ESTScan2.html) to predict for "CDS" and orientation. With nr annotation, the Blast2GO program (version 2.5.0, http://blast2go.com/b2ghome) was used to classify unigenes to GO terms such as molecular function, biological processes, and cellular 
components [39]. After obtaining GO annotations for all unigenes, WEGO software [40] was used to perform GO function classification for all unigenes and to analyze the distribution of $L$. chinense gene functions at the macro level. Using the KEGG pathway database and nr annotation on KEGG, we could cluster multiple unigenes to the same GO terms and the same KEGG pathway [41].

\section{Identifying sequences of phenylpropanoids genes}

Genes in the phenylpropanoid biosynthesis pathway were identified using Illumina sequencing data. They were searched for using a functional annotation file based on the candidate gene name. Following this, each search sequence was further performed by using the BLAST program in the National Center for Biotechnology Information GenBank database (http://www.ncbi.nlm.nih.gov/ BLAST).

\section{CDNA synthesis and real-time PCR}

After total RNA of different organs were extracted, the quality and concentration of different total extracted RNA were evaluated by $1 \%$ agarose gel electrophoresis and spectrophotometric analysis, respectively. For firststrand cDNA synthesis, $1 \mu \mathrm{g}$ of high-quality total RNA was used for reverse transcription (RT) with a ReverTra Ace-kit (Toyobo Co. Ltd., Osaka, Japan). A 20-fold dilution of $20 \mu \mathrm{L}$ of the resulting cDNA was used as a template for quantitative real-time PCR.

Based on the sequences of LcPAL, LcC4H, Lc4CL, LcCHS, LcCHI, LcF3H, LcFLS, LcF3'H, Lc3GT, LcC3H and $L c C O M T$, real-time PCR primers were designed by the Primer 3 website (http://frodo.wi.mit.edu/primer3/) (Additional file 2). The expression of these genes was calculated by relative quantification method with rhe L.chinense Actin housekeeping gene, which was also isolated through NGS sequences (data not shown), as a reference. For quantification of the standard, PCR products amplified from cDNA were purified, and the concentration of the products was measured in order to calculate the number of cDNA copies. Real-time PCR reaction were performed in a $20 \mu \mathrm{L}$ reaction mixture including $5 \mu \mathrm{L}$ of template cDNA, $10 \mu \mathrm{L}$ of $1 \times$ SYBR Green Real-time PCR Master Mix (Toyobo, Osaka, Japan), $0.5 \mu \mathrm{L}$ of each primer $(10 \mu \mathrm{L})$ and DEPC-treated water. Thermal cycling conditions were as follows: $95^{\circ} \mathrm{C}$ for $5 \mathrm{~min}$ and 40 cycles of $95^{\circ} \mathrm{C}$ for $15 \mathrm{~s}, 56^{\circ} \mathrm{C}$ for $15 \mathrm{~s}$, and $72^{\circ} \mathrm{C}$ for $20 \mathrm{~s}$. The PCR reactions were performed on a CFX96 Real-Time system (Bio-Rad Laboratories, Hercules, CA). PCR products were analyzed with the Bio-Rad CFX Manager 2.0 software. Three replications for each sample were used for the real-time PCR analysis. Values were expressed as means \pm SDs.

\section{High performance liquid chromatography analysis}

Different organs of $L$. chinense were freeze-dried at $-80^{\circ} \mathrm{C}$ at $48 \mathrm{~h}$, and then ground into a fine powder using a mortar and pestle. Phenylpropanoids were released from the L.chinense samples $(0.02 \mathrm{~g})$ by adding $3 \mathrm{~mL}$ of methanol containing $0.1 \%$ ascorbic acid $(\mathrm{w} / \mathrm{v})$ at $60^{\circ} \mathrm{C}$ for $1 \mathrm{~h}$. After centrifuging $(3000 \mathrm{rpm})$ the extract, the supernatant was filtered with a $0.22 \mu \mathrm{m}$ Acrodisc syringe filter (Pall Corp.; Port Washington, NY), and then analyzed by HPLC. The phenylpropanoids were separated on C18 column (250× $4.6 \mathrm{~mm}, 5 \mu \mathrm{m}$; RStech, Daejeon, Korea) by an Agilent 1100 HPLC system (Agilent Technologies France, Massy, France) that was equipped with a photodiode array detector. The mobile phase consisted of methanol, water and $0.2 \%$ acetic acid, and the column was maintained at $30^{\circ} \mathrm{C}$. The flow rate was maintained at $1.0 \mathrm{~mL} / \mathrm{min}$, the injection volume was $20 \mu \mathrm{L}$, and the detection wavelength was $280 \mathrm{~nm}$. The concentrations of phenylpropanoid compounds were determined by using a standard curve. All samples were analyzed in triplicate. Values were expressed as means \pm SDs.

\section{Additional files}

\section{Additional file 1: Pathway assignment based on KEGG. \\ Additional file 2: Primers used for real-time PCR.}

\section{Competing interest}

The authors declare that they have no competing interest.

\section{Authors' contributions}

Conceived and designed the experiments: $\mathrm{CHL}$, SUP. Performed the experiments: SZ, PAT, and XL. Analyzed the data: SZ, YBK, HRK, CGP, and JY Wrote the paper: SZ, CHL, and SUP. All authors read and approved the final manuscript.

\section{Acknowledgement}

This work was carried out with the support of "Cooperative Research Program for Agriculture Science \& Technology Development (Project No. PJ906938)" Rural Development Administration, Republic of Korea, and "National High Technology Research and Development Program of China (2011AA100202)".

\section{Author details}

${ }^{1}$ Department of Crop Science, Chungnam National University, 99 Daehak-ro, Yuseong-gu, Daejeon 305-764, Korea. ${ }^{2}$ Green Bio Research Center, Cabbage Genomics Assisted Breeding Supporting Center, Korea Research Institute of Bioscience and Biotechnology (KRIBB), Daejeon 305-806, Korea. ${ }^{3}$ National Institute of Horticultural and Herbal Science, RDA, Bisanro 92, Eumseong, Chungbuk, 369-873, Korea. ${ }^{4}$ State Key Laboratory of Forest Genetics and Tree Breeding, Northeast Forestry University, 26 Hexing Road, Harbin 150040, China.

Received: 31 August 2013 Accepted: 13 November 2013

Published: 19 November 2013

\section{References}

1. Vogt T: Phenylpropanoid Biosynthesis. Mol Plant 2010, 3(1):2-20.

2. Chabannes M, Ruel K, Yoshinaga A, Chabbert B, Jauneau A, Joseleau JP, Boudet AM: In situ analysis of lignins in transgenic tobacco reveals a differential impact of individual transformations on the spatial patterns of lignin deposition at the cellular and subcellular levels. Plant J 2001, 28(3):271-282 
3. Xie YJ, Xu DK, Cui WT, Shen WB: Mutation of Arabidopsis HY1 causes UV-C hypersensitivity by impairing carotenoid and flavonoid biosynthesis and the down-regulation of antioxidant defence. J Exp Bot 2012, 63(10):3869-3883.

4. Widyarini S, Allanson M, Gallagher NL, Pedley J, Boyle GM, Parsons PG, Whiteman DC, Walker C, Reeve VE: Isoflavonoid photoprotection in mouse and human skin is dependent on metallothionein. J Invest Dermatol 2006, 126(1):198-204

5. Parmar N, Dadarwal KR: Stimulation of nitrogen fixation and induction of flavonoid-like compounds by rhizobacteria. J Appl Microbiol 1999, 86 (1):36-44.

6. Kataoka I, Beppu K: UV irradiance increases development of red skin color and anthocyanins in 'Hakuho' peach. Hortscience 2004, 39(6):1234-1237.

7. Hemaiswarya S, Doble M: Combination of phenylpropanoids with 5-fluorouracil as anti-cancer agents against human cervical cancer (HeLa) cell line. Phytomedicine 2013, 20(2):151-158.

8. Pastore S, Mariani V, Cesareo E, Korkina L: Anti-inflammatory effects of phenylpropanoids on human keratinocytes. Free Radical Res 2007, 41:S46.

9. Oh YC, Cho WK, Im GY, Jeong YH, Hwang YH, Liang C, Ma JY: Anti-inflammatory effect of Lycium Fruit water extract in lipopolysaccharide-stimulated RAW 264.7 macrophage cells. Int Immunopharmacol 2012, 13:181-189.

10. Gan L, Zhang SH, Yang XL, Xu HB: Immunomodulation and antitumor activity by a polysaccharide-protein complex from Lycium barbarum. Int Immunopharmacol 2004, 4(4):563-569.

11. Mao F, Xiao BX, Jiang Z, Zhao JW, Huang X, Guo JM: Anticancer effect of Lycium barbarum polysaccharides on colon cancer cells involves G0/G1 phase arrest. Med Oncol 2011, 28(1):121-126.

12. Luo Q, Cai YZ, Yan J, Sun M, Corke H: Hypoglycemic and hypolipidemic effects and antioxidant activity of fruit extracts from Lycium barbarum. Life Sci 2004, 76(2):137-149.

13. Chang RCC, So KF: Use of anti-aging herbal medicine, Lycium barbarum against aging-associated diseases. What do we know so far? Cell Mol Neurobiol 2008, 28(5):643-652.

14. Youn UJ, Kil YS, Kang U, Lee YJ, Shin HJ, Nam JW, Han AR, Sung SH, Kim J, Lee $\mathrm{SM}$, et al: Pyrrole alkaloids from the fruits of Lycium chinense and their hepatoprotective activity. Planta Med 2012, 78(11):1183-1184.

15. Bunghez IR, Marius AS, Marian N, Georgeta R, Rodica-Mariana I: Obtaining of Carotenoid Extract from Lycium Chinense and Characterization Using Spectometrical Analysis. Dig J Nanomater Bios 2012, 7(2):523-528.

16. Zhang JX, Guan SH, Feng RH, Wang Y, Wu ZY, Zhang YB, Chen XH, Bi KS, Guo DA: Neolignanamides, Lignanamides, and Other Phenolic Compounds from the Root Bark of Lycium chinense. J Nat Prod 2013, 76(1):51-58.

17. Shin YG, Cho KH, Kim JM, Park MK, Park JH: Determination of betaine in Lycium chinense fruits by liquid chromatography-electrospray ionization mass spectrometry. J Chromatogr A 1999, 857(1-2):331-335.

18. Fukuda T, Yokoyama J, Ohashi H: Phylogeny and biogeography of the genus Lycium (Solanaceae): Inferences from chloroplast DNA sequences. Mol Phylogenet Evol 2001, 19(2):246-258.

19. Li X, Thwe AA, Park NI, Suzuki T, Kim SJ, Park SU: Accumulation of Phenylpropanoids and correlated gene expression during the development of Tartary Buckwheat Sprouts. J Agr Food Chem 2012, 60(22):5629-5635.

20. Glenn TC: Field guide to next-generation DNA sequencers. Mol Ecol Resour 2011, 11(5):759-769.

21. Mardis ER: Next-generation DNA sequencing methods. Annu Rev Genomics Hum Genet 2008, 9:387-402.

22. Xia J, Wang Q, Jia P, Wang B, Pao W, Zhao Z: NGS catalog: a database of next generation sequencing studies in humans. Hum Mutat 2012, 33(6):E2341-E2355

23. Wan LL, Han J, Sang M, Li AF, Wu H, Yin SJ, Zhang CW: De Novo Transcriptomic Analysis of an Oleaginous Microalga: pathway description and gene discovery for production of next-generation Biofuels. PloS one 2012, 7(4):e35142.

24. Kuang Q, Li LF, Peng JZ, Sun SL, Wang XJ: Transcriptome Analysis of Gerbera hybrida Ray Florets: putative genes associated with Gibberellin metabolism and signal transduction. PloS one 2013, 8(3):e57715.

25. Chang L, Chen JJ, Xiao YM, Xia YP: De novo characterization of Lycoris sprengeri transcriptome using Illumina GA II. Afr J Biotechnol 2011, 10(57):12147-12155
26. Lois R, Dietrich A, Hahlbrock K, Schulz W: A phenylalanine ammonia-lyase gene from parsley: structure, regulation and identification of elicitor and light responsive cis-acting elements. Embo J 1989, 8(6):1641-1648.

27. Shang QM, Li L, Dong CJ: Multiple tandem duplication of the phenylalanine ammonia-lyase genes in Cucumis sativus L. Planta 2012, 236(4):1093-1105.

28. Tuan PA, Park NI, Li X, Xu H, Kim HM, Park SU: Molecular Cloning and Characterization of Phenylalanine Ammonia-lyase and Cinnamate 4-Hydroxylase in the Phenylpropanoid Biosynthesis Pathway in Garlic (Allium sativum). J Agr Food Chem 2010, 58(20):10911-10917.

29. Tuan PA, Park WT, Xu H, Park NI, Park SU: Accumulation of Tilianin and Rosmarinic Acid and Expression of Phenylpropanoid Biosynthetic Genes in Agastache rugosa. J Agr Food Chem 2012, 60(23):5945-5951.

30. Park NI, Li XH, Suzuki T, Kim SJ, Woo SH, Park CH, Park SU: Differential Expression of Anthocyanin Biosynthetic Genes and Anthocyanin Accumulation in Tartary Buckwheat Cultivars 'Hokkai T8' and 'Hokkai T10'. J Agr Food Chem 2011, 59(6):2356-2361.

31. Li X, Kim YB, Kim Y, Zhao S, Kim HH, Chung E, Lee JH, Park SU: Differential stress-response expression of two flavonol synthase genes and accumulation of flavonols in tartary buckwheat. J Plant Physiol 2013, 170(18):1630-1636.

32. Dillard CJ, German JB: Phytochemicals: nutraceuticals and human health. J Sci Food Agr 2000, 80(12):1744-1756.

33. Sakagami H, Asano K, Satoh K, Takahashi K, Terakubo S, Shoji Y, Nakashima H, Nakamura W: Anti-stress activity of mulberry juice in mice. In vivo 2006 20(4):499-504.

34. Du J, He ZD, Jiang RW, Ye WC, Xu HX, But PPH: Antiviral flavonoids from the root bark of Morus alba L. Phytochemistry 2003, 62(8):1235-1238.

35. Dong JZ, Wang Y, Wang SH, Yin LP, Xu GJ, Zheng C, Lei C, Zhang MZ: Selenium increases chlorogenic acid, chlorophyll and carotenoids of Lycium chinense leaves. J Sci Food Agr 2013, 93(2):310-315.

36. Hartikainen $\mathrm{H}$ : Biogeochemistry of selenium and its impact on food chain quality and human health. J Trace Elem Med Bio 2005, 18(4):309-318.

37. Bao Z, Guo J, Zhou W, Zhang Y, He Z, Hu L: Optimization of tissue culture regeneration system of lycium barbarum. J Northwest Forest Univ 2010, 25(5):73-76

38. Grabherr MG, Haas BJ, Yassour M, Levin JZ, Thompson DA, Amit I, Adiconis X, Fan L, Raychowdhury R, Zeng Q, et al: Full-length transcriptome assembly from RNA-Seq data without a reference genome. Nat Biotechnol 2011, 29(7):644-652

39. Conesa A, Gotz S, Garcia-Gomez JM, Terol J, Talon M, Robles M: Blast2GO: a universal tool for annotation, visualization and analysis in functional genomics research. Bioinformatics 2005, 21(18):3674-3676.

40. Ye J, Fang L, Zheng HK, Zhang Y, Chen J, Zhang ZJ, Wang J, Li ST, Li RQ Bolund L, et al: WEGO: a web tool for plotting GO annotations. Nucleic Acids Res 2006, 34:W293-W297.

41. Kanehisa M, Goto S: KEGG: Kyoto Encyclopedia of Genes and Genomes. Nucleic Acids Res 2000, 28(1):27-30.

doi:10.1186/1471-2164-14-802

Cite this article as: Zhao et al:: Identification of phenylpropanoid biosynthetic genes and phenylpropanoid accumulation by transcriptome analysis of Lycium chinense. BMC Genomics 2013 14:802.

\section{Submit your next manuscript to BioMed Central and take full advantage of:}

- Convenient online submission

- Thorough peer review

- No space constraints or color figure charges

- Immediate publication on acceptance

- Inclusion in PubMed, CAS, Scopus and Google Scholar

- Research which is freely available for redistribution 